\title{
The smart respiratory monitoring integrated pulmonary index: An overview of the literature
}

\author{
Akıllı solunum monitörizasyonu entegre pulmoner indeks: \\ Literatüre genel bir bakış
}

\author{
Cengiz KAYA
}

\begin{abstract}
Assessments of the respiratory functions of patients are frequently based on measurements of arterial oxygen saturation $\left(\mathrm{SpO}_{2}\right)$, end-tidal carbon dioxide $\left(\mathrm{PetCO}_{2}\right)$, respiratory rate $(\mathrm{RR})$, and heart rate (HR). Interpreting the data derived from the measurements of these parameters can be highly problematic for health care workers, especially those who are inexperienced in monitoring the respiratory functions. However, the integrated pulmonary index (EPI) monitorization can offer a single value for easy evaluation of ventilation and oxygenization. This method combines the $\mathrm{SpO}_{2}$, PetCO $\mathrm{2}, \mathrm{RR}$, and $\mathrm{HR}$ values in a mathematical model to obtain an index value, EPI. This value can be continuously monitored as either a numerical value or a waveform displayed on the monitor. In reviewing the literature on EPI, we noted that the EPI has been used more frequently in procedures performed under sedation, such as gastroscopy, colonoscopy, cardioversion, and tooth extraction, and in intensive care units, for adjusting settings and modes of mechanical ventilation, evaluating the compliance of the patient to the mechanical ventilation therapy, and monitoring the weaning process, and during the postoperative follow-up of patients who have undergone coronary bypass surgery, to detect respiratory problems and evaluate the weaning process. In conclusion, using the EPI to monitor respiratory function has attracted considerable attention because it ensures noninvasive, dynamic, and real-time measurements, reflects the respiratory states of patients with higher specificity, and sensitive, and enables earlier recognition of respiratory problems. In addition, an important advantage of this system is that it allows physicians, in particular those who are not anesthesiologists, to use the EPI index to evaluate the respiratory states of patients rapidly and easily.
\end{abstract}

Keywords: Integrated pulmonary index, patient monitoring, sedation, endoscopy öz

Hastanın solunum fonksiyonlarını değerlendirmek için sıklıkla arteriyel oksijen satürasyonu $\left(\mathrm{SpO}_{2}\right)$, end-tidal karbondioksit $\left(\mathrm{PetCO}_{2}\right)$, solunum sayısı (SS) ve kalp hızı (KH) parametreleri kullanılmaktadır. Bu parametrelerden elde edilen verilerin yorumlanması özellikle solunum monitörizasyonu konusunda deneyimli olmayan sağlık çalışanları için önemli bir sorun oluşturur. Entegre pulmoner indeks (EPI) monitörizasyonu ise ventilasyon ve oksijenasyonu kolayca değerlendirebilmek için tek bir değer sunabilmektedir. Bu yöntemde $\mathrm{SpO}_{2^{\prime}}$ PetCO $\mathrm{CO}_{2^{\prime}} \mathrm{SS}$ ve $\mathrm{KH}$ değerleri matematiksel bir modelde kombine edilerek bir indeks değer - EPI - elde edilir. Bu indeks değer monitörde sayısal veri ya da bir dalga formu şeklinde devamlı olarak takip edilebilmektedir. EPi literatüre bakıldığında, daha çok sedasyon altında yapılan işlemlerde (gastroskopi, kolonoskopi, kardiyoversiyon, diş çekimi gibi), yoğun bakımlarda (mekanik ventilatör ayarlarının yapılması, ventilatör/hasta uyumunun değerlendirilmesi, weaning sürecinin takibi için) ve koroner arter bypass greft cerrahisi uygulanan hastaların postoperatif takibinde (solunumsal problemlerin tespit edilmesi ve weaning sürecinin değerlendirilmesi için) kullanıldığını görmekteyiz. Sonuç olarak, EPi monitörizasyonu, noninvaziv, dinamik ve gerçek zamanlı bir ölçüme olanak vermesi, solunum durumunu yüksek spesifite ve sensitivite ile yansıtması ve solunum sorunlarının daha erken dönemde fark edilmesini sağlayabilmesi nedeniyle dikkatleri üzerine çekmiştir. Ayrıca özellikle anestezist olmayan hekimlerin hastanın solunum durumunu kolay ve hızlı bir şekilde değerlendirmesine olanak vermesi bu yönteme önemli bir avantaj sağlamaktadır.

Anahtar kelimeler: Entegre pulmoner indeks, hasta monitörizasyonu, sedasyon, endoskopi

\section{Giriş}

Anestezi uygulamalarında hastanın solunum fonksi- yonunun doğru olarak değerlendirilmesi büyük önem gösterir. Solunum sayısı (SS) ve arteriyel oksijen satürasyonu $\left(\mathrm{SpO}_{2}\right)$ solunum fonksiyonunun değerlendi-

Received: 18.10 .2016

Accepted: 15.11.2016

Department of Anesthesiology, Ondokuz Mayis University, Samsun, Turkey

Yazışma adresi: Cengiz Kaya, Department of Anesthesiology, Ondokuz Mayis University, Samsun, Turkey

e-mail: raufemre@yahoo.com 
rilmesinde tek başına yeterli olmamaktadır ${ }^{1}$. Bunlara ilave olarak kapnograf ile end-tidal karbondioksit $\left(\mathrm{PetCO}_{2}\right)$ değerlerinin takip edilmesi, solunumun değerlendirilmesine önemli katkıda bulunmuştur ${ }^{2,3}$.

Monitördeki verilerin doğru olarak değerlendirilmesi için hasta takibini yapan sağılı çalışanlarının sürekli bir eğitime tabii tutulması gerekmektedir ${ }^{4}$. Yapılan bir çalışmada, monitörde üçten fazla parametrenin eşzamanlı olarak değerlendirilmesinin zor olduğu ve yanlış yorumlamalara neden olabileceği bildirilmektedir $^{5}$. Ventilasyon ve oksijenasyonu kolayca değerlendirebilmek için tek bir değer sunan entegre pulmoner indeks (EPI) gibi bir monitörizasyon tekniği bu sorunları azaltacaktır ${ }^{4}$. Bu monitörizasyon yönteminde $\mathrm{SpO}_{2}, \mathrm{PetCO}_{2}$, SS ve kalp hızı $(\mathrm{KH})$ değerleri matematiksel bir modelde kombine edilerek bir indeks değer - EPi - elde edilmektedir. Bu indeksin monitörde bir sayısal değer ya da bir dalga formu şeklinde devamlı olarak takibi olası olmaktadır. EPi değeri 1 (solunum yetmezliği) ile 10 (normal solunum) arasında bir ölçekte değerlendirilir (Tablo 1). Böylece sağlık çalışanının deneyimine bakılmaksızın, hastanın solunum durumunun kolay ve hızlı bir şekilde değerlendirilebilmesi ve oluşan sorunlara erken dönemde müdahale edebilmesi olası olabilmektedir ${ }^{4,6,7}$.

Tablo 1. EPi skorlaması.

\begin{tabular}{ll}
\hline EPi Skoru & Hastanın Durumu \\
\hline 10 & Normal \\
$8-9$ & Normal sınıra yakın \\
7 & Normal sınıra yakın ancak dikkatli olunmalı. \\
$5-6$ & Dikkatli olunmalı, müdahale gerekebilir. \\
$3-4$ & Müdahale edilmeli. \\
$1-2$ & Derhal müdahale edilmeli. \\
\hline
\end{tabular}

\section{EPi Algoritması:}

EPi değerleri elde edilirken kullanılan algoritma için özel bir matematik model (Fuzzy Logic Mathematical Model) kullanılmaktadır ${ }^{8}$. Bu model insan düşünce sistemini taklit eder (yapay zeka) ve değişkenleri belirli olasılıklar üzerinden değerlendirilerek sonuca ulaşır ${ }^{9}$. Algoritma ile ilgili ilk çalışma, Taft ve ark. ${ }^{6}$ tarafından yapıldı. Bu çalışmada, hemşireler, fizyologlar, anestezistler ve solunum terapistlerinden oluşan 18 kişilik bir ekiple 85 hastaya ait dört parametre $\left(\mathrm{SpO}_{2}\right.$, $\mathrm{PetCO}_{2}, \mathrm{SS}, \mathrm{KH}$ ) değerlendirildi. Daha sonra Ronen ve ark. ${ }^{4}$ tarafından solunum monitörizasyonu konusunda deneyimli 22 klinisyen ile (hemşireler, doktorlar, anestezistler ve solunum terapistleri) 235 hastadan alınan verilerin 85 adet kombinasyonu oluşturuldu. $\mathrm{Bu}$ kombinasyonlar oluşturulurken, $\mathrm{SpO}_{2}$ ve $\mathrm{KH}$ parametreleri için yüksek, normal, düşük; $\mathrm{PetCO}_{2}$ ve $\mathrm{SS}$ için ise çok yüksek, yüksek, normal, düşük, çok düşük değerleri tanımlandı. Ayrıca olasılıklar oluşturulurken, "Eğer-O zaman", "Veya” ve "Ve" sözcükleri kullanıldı. Böylece bir algoritma çizelgesi oluşturuldu. Buna göre örneğin, "eğer PetCO 2 ve SS çok yüksek ve $\mathrm{SpO}_{2}$ normal ve $\mathrm{KH}$ yüksek ise o zaman EPI ikidir" veya "eğer $\mathrm{PetCO}_{2}$ normal veya yüksek ve SS normal ve $\mathrm{SpO}_{2}$ ve $\mathrm{KH}$ normal ise o zaman EPI sekizdir" gibi EPI değerleri tespit edildi. Aynı metodoloji kullanılarak pediatrik yaş grupları içinde EPi değerleri elde edildi. Pediatrik yaş grubunda normal vital değerler yaşa bağımlı olarak değiştiğinden bu hastalar için 1-3 yaş, 3-6 yaş ve 6-12 yaş olarak üç grup belirlendi. Bir yaş altında ise kilo ve gestasyonel yaşa bağımlı olarak normal vital değerler arasında büyük değişiklikler olduğu için bu hastalar değerlendirme dışında bırakıldı.

\section{Algoritmanın Validitesi:}

Algoritma oluşturulurken, ayrıca geçerliliği ile ilgili olarak geriye dönük 523 hasta değerlendirildi. Solunum monitörizasyonu konusunda deneyimli aynı ekip, $\mathrm{SpO}_{2}, \mathrm{PetCO}_{2}$, SS, $\mathrm{KH}$ parametrelerini göz önüne alarak ciddi (apne, ciddi hipoksi) ve daha hafif düzeyde (bradipne, hipoventilasyon, hipoksi gibi) solunum sıkıntısı olan hastaları tespit etmeleri istendi. Aynı zamanda tüm hastalar EPI algoritması ile de değerlendirildi. Sonuçta, EPi algoritmasının solunumsal sorunları tespit etmede yüksek sensitivite (\%83-100) ve spesifiteye (\%74-96) sahip olduğu görüldü. Dört Sedasyon uygulanan 76 pediatrik olgunun değerlendirildiği bir çalışmada ise, EPI algoritmasının sensitivitesi $\% 100$ ve spesifitesi \%98 olarak bulundu ${ }^{10}$. Başka bir çalışmada, sedasyon altında endoskopik girişim planlanan 109 çocuk hasta değerlendirildi. Klinik ola- 
rak önemli kabul edilen solunum sorunları (apne, hipoksi, bradipneik hipoventilasyon+hipoksi, hipopneik hipoventilasyon+hipoksi) EPI monitörü tarafından $\% 97$ sensitivite ve $\% 89$ spesifite ile tespit edildi ${ }^{11}$.

\section{Klinik Kullanım}

Sedasyon uygulanan hastalarda ilaçlara bağlı olarak görülebilen solunum depresyonu ve hava yolu obstrüksiyonu önemli bir morbidite nedenidir ${ }^{12}$. Bu nedenle hastalar hem görsel olarak hem de fizyolojik parametreleri ölçebildiğimiz cihazlarla takip edilme$\operatorname{lidir}^{13}$. Bu hastalarda özellikle ilave oksijen desteği de uygulandığında tek başına pulse oksimetri ile alveoler hipoventilasyonun zamanında fark edilmesi güç olmaktadır ${ }^{2,3}$. Bu yüzden sedasyon uygulamalarında arteriyel oksijenasyonu monitörize etmek için pulse oksimetri ve ventilasyonu değerlendirmek için de kapnografın birlikte kullanılması önerilmektedir ${ }^{1,14-19}$. EPI monitörizasyonu ise pulse oksimetri ve kapnograf değerleriyle fizyolojik parametreleri (SS ve $\mathrm{KH}$ ) birleştirerek tek bir değer sunmaktadır. Ayrıca Amerika Birleşik Devletleri Gıda ve İlaç Dairesi (FDA), EPi monitörlerinin sedasyon uygulanan hastalarda solunum parametrelerindeki değişiklikleri takip etmek amacıyla kullanılabileceğini belirtmektedir ${ }^{20}$.

EPI değerlerinin, sedasyon uygulanan hastalarda oluşan solunum sorunlarını belirlemedeki etkinliğini değerlendirmek için yapılan bir çalışmada, kolonoskopi yapılacak 51 hasta değerlendirildi ${ }^{20}$. İşlemler esnasında 113 solunumsal sorun (en az $1 \mathrm{dk}$. boyunca $\mathrm{SpO}_{2}$ $\leq 92 \%$ ve/veya $\mathrm{SS} \leq 8$ ve/veya $\mathrm{PetCO}_{2} 20 \%$ azalma) tespit edilmesine rağmen, EPI monitörü tarafından ancak bunların \%42,9'u belirlenebildi. Yazarlar bu sonuç üzerine EPI monitörünün klinik deneyimi olmayan sedasyon uygulayıcılarını yanıltabileceğini ve EPI algoritmasının gözden geçirilmesinin gerektiğini vurguladılar. Başka bir çalışmada ise, sedasyon altında gastroskopi/kolonoskopi uygulanan 30 hasta EPi monitörü ile takip edildi. Beş hastada maske ventilasyonu gerekmesine rağmen (EPI skoru 1-3), ancak bu hastaların yalnızca ikisinde $\mathrm{SpO}_{2}$ değerleri düşük bulundu. Yazarlar tarafından EPI monitörizasyonun pulse oksimetriye göre daha erken uyarı yaparak hasta güvenliğini sağlamakta anesteziste yardımcı olabileceği rapor edildi ${ }^{21}$. Yine başka bir çalışmada, sedasyon eşliğinde elektriksel kardiyoversiyon uygulanan 45 hasta değerlendirildi ve EPI monitörizasyonunun respiratuvar parametrelerin takibinde güvenli ve etkili olduğu bulundu ${ }^{22}$. Koshika ve ark.'nın ${ }^{23}$ yaptıkları çalışmada ise, sedasyon altında maksillofasiyal cerrahi (diş çekme veya dental implantasyon gibi) uygulanan 100 hasta değerlendirildi ve tüm hipopne ve apne ataklarını EPi monitörü tarafından tespit edebildiği görüldü. Ayrıca EPI değerinin aniden ikinin altına düşmesinin apne için önemli bir gösterge olduğu belirtildi.

Gozal ve ark. ${ }^{10}$ ise derin sedasyon uygulanan çocuklarda (yaş ortalaması 4,8) yaptıkları çalışmada, EPi monitörünün solunum sorunlarını (hipoksi, hiperkapni, apne) tespit etmedeki güvenilirliğini değerlendirdiler. Sonuçta, EPI mönitörünün tüm solunum sorunlarını tespit edebildiği gözlendi (spesifitesi \%98). Yazarlar tarafından derin sedasyon uygulanan pediatrik hastaların takibinde EPI monitörünün özellikle deneyimi az olan sağlık çalışanları için yararlı olabileceği belirtildi. Başka bir çalışmada, sedasyon eşliğinde gastrointestinal endoskopi uygulanan çocuklarda (yaş ortalaması 8,6 $\pm 5,0$ ) endoskopiyi yapan doktorların klinik değerlendirmesi ile EPI değerleri karşılaştırıldığında sonuçların birbirine uyumlu olduğu görüldü. Sonuç olarak, endoskopi yapan hekimler için hasta takibinde EPI monitörizasyonun güvenli ve yararlı olabileceğini belirtildi ${ }^{7}$. Yine başka bir çalışmada 109 endoskopi uygulanan çocuk hasta (1-18 yaş) değerlendirildi. İşlem süresince meydana gelen tüm apne ve hipoksi atakları EPI monitörü tarafından tespit edilirken (sensitivite $\% 100$, spesifite $\% 98$ ), pulse oksimetri tarafından yalnızca hipoksi atakları belirlenebildi ${ }^{11}$. Tüm bu sonuçlara bakıldığında EPI monitörizasyonu, sedasyon uygulanan hastaların solunum durumunu takip etmede yararlı olabilir düşüncesindeyiz.

Oglesby ve ark. ${ }^{24}$ tarafından "EPI monitörizasyonu, mekanik ventilasyon uygulanan yoğun bakım hastalarında mekanik ventilasyon etkinliğinin değerlendi- 
rilmesinde ve ekstübasyon zamanın belirlenmesinde yardımcı olabilir" hipotezi ile bir çalışma planlandı. Bu çalışmada 45 gün boyunca mekanik ventilasyon uygulanan dört hastaya ait 267 adet veri [EPI değerleri, mekanik ventilatör/hasta uyumu ve hastadaki klinik değişiklikler (respiratuvar asidoz gibi)] değerlendirildi. Sonuç olarak, mekanik ventilatör/hasta uyumunun iyi olduğu dönemlerde EPI değerlerinin yüksek olduğu, hastanın durumunun kötüleştiği dönemlerde (respiratuvar asidoz gibi) ise EPi değerlerinin düşük olduğu gözlendi. Yazarlar tarafından optimal mekanik ventilatör ayarlarının belirlenmesinde ve ekstübasyonun daha erken yapılabilmesinde EPI monitörizasyonunun yararlı olabileceği belirtildi. Başka bir çalışmada, 21 yoğun bakım hastasında EPI değerleri ile arteryel kan gazı değerleri karşılaştırıldı. Sonuçta, EPi değerleri ile arteryel kan gazının gösterdiği solunum durumu ve $\mathrm{SaO}_{2}$ arasında doğru orantı ve $\mathrm{PaCO}_{2}$ ile de ters orantı olduğu görüldü. Bunun üzerine EPI monitörizasyonunun yoğun bakım hastalarında solunum durumunu değerlendirmek için kullanılabileceği ve arteryel kan gazı ölçümlerine göre daha dinamik ve eşzamanlı bir ölçüm olanağı sağladığı belirtildi ${ }^{25}$. Başka bir çalışmada ise, yoğun bakımda takip edilen 100 hastanın arteryel kan gazı değerleri ile EPi değerleri arasında bir korelasyon olup olmadığı araştırıldı. Sonuç olarak, EPI monitöründeki $\mathrm{SPO}_{2}$ ve $\mathrm{PetCO}_{2}$ değerleri ile arteryel kan gazındaki $\mathrm{SaO}_{2}$ ve $\mathrm{PaCO}_{2}$ değerleri arasında korelasyon olduğu görüldü. Sonuçta, bu non-invazif, dinamik ölçüm tekniğinin arteryel kan gaz analiz ölçümlerine bir alternatif olarak kullanılabileceği rapor edildi ${ }^{26}$. Kumar ve ark. ${ }^{27}$ tarafından yapılan çalışmada ise, mekanik ventilasyon uygulanan 43 yoğun bakım hastasında ventilatörden ayırma (weaning) başarısı ile EPi değerleri arasında bir ilişki olup olmadığı araştırıldı. Sonuç olarak, weaning işleminin başarılı olduğu hastaların EPi değerleri, başarısız olunan hastalardan daha yüksek bulundu. Tüm bu sonuçlar değerlendirildiğinde, EPI monitörizasyonun mekanik ventilasyon uygulanan yoğun bakım hastalarında mekanik ventilasyonun etkinliğini ve weaning sürecini değerlendirilmede yardımcı olabileceği düşünüldü.
Kuzkov ve ark. ${ }^{28}$ tarafından yapılan çalışmada, koroner arter bypass greft cerrahisi (off-pump) uygulanan 33 hastanın postoperatif (12 saat) EPI skorları değerlendirildi. Sonuçta, EPi monitörizasyonunun erken postoperatif dönemdeki respiratuvar sorunları tespit etmede ve ekstübasyonun başarılı olup olamayacağını belirlemede yararlı olabileceği gösterildi. Yine başka bir çalışmalarında, koroner arter bypass greft cerrahisi (off-pump) uygulanan 72 hastanın weaning sürecinin takibinde EPI monitörizasyonunun bir katkısı olup olmadığı araştırıldı. Sonuç olarak, EPi monitörizasyonunun bu sürecin değerlendirilmesinde faydalı olabileceği belirtildi ${ }^{29}$. Kumar ve ark.'nın ${ }^{30}$ yaptığı bir çalışmada ise, koroner arter bypass greft cerrahisi uygulanan 24 obez hastanın weaning süreci EPi monitörizasyonu ile değerlendirildi. Weaning işleminin başarılı olduğu hasta grubunda EPi skorları daha yüksek bulundu.

Literatüre bakıldığında, EPI monitörizasyonun daha çok sedasyon altında yapılan işlemlerde (gastroskopi, kolonoskopi, kardiyoversiyon, diş çekimi gibi), yoğun bakımlarda (mekanik ventilatör ayarlarının yapılması, ventilatör/hasta uyumunun değerlendirilmesi, weaning sürecinin takibi için) ve koroner arter bypass greft cerrahisinin postoperatif döneminde (solunumsal sorunların tespit edilmesi ve weaning sürecinin değerlendirilmesi için) kullanıldığı görülmektedir. Özellikle anestezist olmayan hekimlerin hastanın solunum durumunu değerlendirirken, bu dört parametreyi aynı anda değerlendirip yorumlaması güç olmaktadır. Ayrıca bu fazla veri yükü ve solunum monitörizasyonu konusunda yeterli deneyime sahip olmama verilerden yanlış sonuçlar çıkarılmasına neden olabilir. EPi monitörizasyonu bu hekim grubunun verileri kolay ve hızlı bir şekilde değerlendirmesine olanak sağlayarak önemli bir avantaj elde etmektedir. Sonuç olarak, EPI monitörizasyonu non-invazif, dinamik, gerçek zamanlı ve devamlı ölçüm sağlayan bir yöntem olması, solunum durumunu yüksek spesifite ve sensitivite ile yansıtması ve sayısal tek bir değer ile sorunlarının daha erken dönemde fark edilmesini sağlayabilmesi nedeniyle dikkatleri üzerine çekmektedir. 


\section{KAYNAKLAR}

1. Stoelting RK, Overdyk FJ. Recommendations-Conference on Electronic Monitoring Strategies. 2015; http://www.apsf. org/announcements. php?id=7.

2. D'Arcy Y. Turning the tide on respiratory depression. Nursing 2013;43(9):38-45. https://doi.org/10.1097/01.NURSE.0000432909.39184.e1

3. Dar AQ, Shah ZA. Anesthesia and sedation in pediatric gastrointestinal endoscopic procedures: A review. World J Gastrointest Endosc 2010;2(7):257-262. https://doi.org/10.4253/wjge.v2.i7.257

4. Ronen M, Weissbrod R, Overdyk FJ, Ajizian S. Smart respiratory monitoring: clinical development and validation of the $\mathrm{IPI}^{\mathrm{TM}}$ (Integrated Pulmonary Index) algorithm. Journal of Clinical Monitoring and Computing. 2016. https://doi.org/10.1007/s10877-016-9851-7

5. Dawant BM. Knowledge-based systems for intelligent patient monitoring and management in critical care environments. Biomedical Engineering Handbook. CRC Press Ltd. 2000;208.

6. Taft A, Ronen M, Epps C, et al. A Novel Integrated Pulmonary Index (IPI) Quantifies Heart Rate, EtcO 2 , Respiratory Rate and $\mathrm{SpO}_{2} \%$. Anesthesiology 2008;(A1682):109.

7. Lightdale JR, Fredette ME, Atmadja ML, et al. M1563: Pilot Study of the Smart Capnography Integrated Pulmonary Index $^{\mathrm{TM}}$ in a Pediatric Gastroenterology Procedure Unit. Gastrointestinal Endoscopy 2010;71(5):AB255.

https://doi.org/10.1016/j.gie.2010.03.564

8. Hines J, Tsoukalas LH, Uhrig RE. MATLAB supplement to Fuzzy and neural approaches in engineering. John Wiley \& Sons, Inc.; 1997.

9. Bates JH, Young MP. Applying fuzzy logic to medical decision making in the intensive care unit. Am J Respir Crit Care Med 2003;167(7):948-952. https://doi.org/10.1164/rccm.200207-777CP

10. David Gozal, Gozal Y. The Integrated Pulmonary Index: Validity, Safety and Application in the Pediatric Population. Annual Meeting of the American Society Anesthesiologists 2009: A390.

11. Garah J, Adiv OE, Rosen I, Shaoul R. The value of Integrated Pulmonary Index (IPI) monitoring during endoscopies in children. J Clin Monit Comput 2015;29(6):773-778. https://doi.org/10.1007/s10877-015-9665-z

12. Practice Guidelines for Sedation and Analgesia by NonAnesthesiologists. Anesthesiology 2002;96(4):1004-1017. https://doi.org/10.1097/00000542-200204000-00031

13. Cohen LB, Delegge MH, Aisenberg J, et al. AGA Institute review of endoscopic sedation. Gastroenterology 2007;133(2):675-701. https://doi.org/10.1053/j.gastro.2007.06.002

14. Jarzyna D, Jungquist CR, Pasero C, et al. American Society for Pain Management Nursing guidelines on monitoring for opioid-induced sedation and respiratory depression. Pain Manag Nurs 2011;12(3):118-145 e110.

15. Fanning RM. Monitoring during sedation given by nonanaesthetic doctors. Anaesthesia 2008;63(4):370-374. https://doi.org/10.1111/j.1365-2044.2007.05378.x

16. Burton JH, Harrah JD, Germann CA, Dillon DC. Does end-tidal carbon dioxide monitoring detect respiratory events prior to current sedation monitoring practices? Acad Emerg Med 2006;13(5):500-504.

https://doi.org/10.1111/j.1553-2712.2006.tb00999.x

17. Deitch K, Miner J, Chudnofsky CR, et al. Does end tidal $\mathrm{CO}_{2}$ monitoring during emergency department procedural sedation and analgesia with propofol decrease the incidence of hypoxic events? A randomized, controlled trial. Ann Emerg Med 2010;55(3):258-264.

https://doi.org/10.1016/j.annemergmed.2009.07.030

18. Qadeer MA, Vargo JJ, Dumot JA, et al. Capnographic monitoring of respiratory activity improves safety of sedation for endoscopic cholangiopancreatography and ultrasonography. Gastroenterology 2009;136(5):1568-1576; quiz 1819-1520.

19. Yarchi D, Cohen A, Umansky T, et al. Assessment of end-tidal carbon dioxide during pediatric and adult sedation for endoscopic procedures. Gastrointest Endosc 2009;69(4):877-882. https://doi.org/10.1016/j.gie.2008.05.054

20. Berkenstadt H, Ben-Menachem E, Herman A, Dach R. An evaluation of the Integrated Pulmonary Index (IPI) for the detection of respiratory events in sedated patients undergoing colonoscopy. Journal of Clinical Monitoring and Computing 2012;26(3):177-181. https://doi.org/10.1007/s10877-012-9357-x

21. Turan G, Tas BA, Demiroluk O, et al. Integrated Pulmonary Index in Endoscopic Procedures on Sedation. Bosphorus Medical Journal 2015;2(3):85-88.

22. Sabbatani $P$, Mantovan R. Electrical cardioversion of atrial fibrillation: Evaluation of sedation safety with midazolam by means of $\mathrm{EtCO}_{2}$ and IPI algorithm analysis. International Journal of Cardiology 2013;169(6):430-432. https://doi.org/10.1016/j.ijcard.2013.10.015

23. Koshika K, Ouchi T, Serita R, Koitabashi T. Impact of Integrated Pulmonary Index (IPI) to Detect Respiratory Depression During Maxillofacial Surgery Under Intravenous Sedation. Annual Meeting of the American Society Anesthesiologists 2015: A4162.

24. Oglesby $H$, Lain D. Do changes in the integrated pulmonary index (IPI) reflect patient/ventilator interaction? preliminary data. Abstracts of the 2013 Annual Meeting of the Society for Technology in Anesthesia (STA). January 9-12, 2013. Scottsdale, Arizona, USA. Anesth Analg 2013;117(4 Suppl).

25. Restrepo RD, Otaibi AA, Wittnebel L, et al. Correlation Between the Integrated Pulmonary Index (IPI ${ }^{\mathrm{TM}}$ ) and Arterial Blood Gases in a Medical-Surgical ICU in Saudi Arabia. Abstracts of the 2013 Annual Meeting of the Society for Technology in Anesthesia (STA). January 9-12, 2013. Scottsdale, Arizona, USA. Anesth Analg 2013;117(4S Suppl.):54-55.

26. Turan G, Kuplay $Y$, Karip C, et al. Integrated Pulmonary Index: A New Strategy for Respiratory Patients Evaluation. International Journal of Anesthetics and Anesthesiology 2016;3(1). https://doi.org/10.23937/2377-4630/3/1/1042

27. Kumar V, Arthur Taft R, Susan Johnson R, et al. Progressive Evaluation of Integrated Pulmonary Index (IPI) During Spontaneous Breathing Trials in Medical and Surgical Patients. Annual Meeting of the American Society Anesthesiologists October 2013: A4237.

28. Kuzkov V, Gaidukov K, Fot E, et al. Integrated pulmonary index reflects respiratory function after elective coronary artery bypass grafting: 5AP4-5. European Journal of Anaesthesiology (EJA) 2011;28:78. https://doi.org/10.1097/00003643-201106001-00247

29. Kuzkov V, Fot E, Gaidukov K, et al. Integrated pulmonary in$\operatorname{dex}(\mathrm{IPI})$ is associated with duration of postoperative respiratory support after coronary artery bypass grafting and body mass index: 5AP4-6. European Journal of Anaesthesiology (EJA) 2012;29:90. https://doi.org/10.1097/00003643-201206001-00292

30. Kumar V, Taft A, Herrington R, et al. 378: Integrated pulmonary index for successful weaning obese patients after cardiac bypass surgery. Critical Care Medicine 2013;41(12):A90. https://doi.org/10.1097/01.ccm.0000439522.13687.5a 